\title{
Isolamento e caracterização \\ da hemoglobina de Brachyplatystoma sp.: um bagre tropical (*)
}

\author{
Joseph P. Martin ('); Maurizio Brunori ${ }^{(2}$ ); Robert L. Garlick ( ${ }^{3}$ ); Dennis A. Powers ( ${ }^{4}$ )
}

\begin{abstract}
Resumo
O componente único da hemoglobina de Brashyplatysoma $\mathrm{sp}$. tem um peso molecular aparente de 69000 determinado por filtração de gel. A hemoglobina apresenta efeito $\mathrm{Bohr}$ tanto ácido quanto básico, efeito de fosfato orgânico e não apresenta efeito Root. $O \mathrm{P}_{1 / 2}$ de oxigênio do sangue completo varia de $10.7 \mathrm{~mm} \mathrm{Hg}$ em soluções equilibradas no ar até $25,1 \mathrm{~mm} \mathrm{Hg}$ após a adição de $5,6 \% \mathrm{CO}_{2}$ ao gás equilibrado. O efeito Bohr medido para hemoglobina "stripped" entre pH 7,0 e 8,0 é $\Delta$ log $\mathrm{P}_{1 / 2} / \Delta \mathrm{p}=\mathrm{H}-0,23$. Adições de $1 \mathrm{mM}$ ATP induzem mudança no efeito Bohr a $\Delta \log \mathrm{P}_{1 / 2} / \Delta \mathrm{pH}=-0,58$ na mesma faixa de $\mathrm{pH}$. O valor $\mathbf{n}$ das soluçōes de hemoglobina "stripped" varia de 1 a pH 7,0. Adições de $1 \mathrm{mM}$ ATP mudam a variação de $\mathbf{n}$ a valores de $\mathrm{pH}$ mais altos causando um aumento no valor de $\mathrm{n}, \mathrm{n}=2$ a pH 7,4. As cinéticas da ligação de monóxido de carbono e dissociação de oxigênio são dependentes do $\mathrm{pH}$. A taxa $\mathrm{CO}$ "on" vira autocatalitica à medida que $\mathrm{opH}$ diminui, indicando interações positivas das subunidades. A taxa $\mathrm{O}_{2}$ "off" foi homogênea a todos os valores do $\mathrm{pH}$. Os efeitos Bohr da hemoglobina de Brachyplatystoma e outras hemoglobinas de pimeloidídeos são maiores que as determinadas para as hemoglobinas não traccionadas de espécies mais sedentárias de outras famílias de bagres, tais como Loricariidae o Callichthyidae.
\end{abstract}

\section{INTRODUÇÃo}

Estudos em hemoglobinas isoladas de numerosas espécies de peixe demonstram amplamente que as hemoglobinas têm evoluído uma larga faixa de propriedades funcionais através de linhas taxonômicas. Existem diferenças entre hemoglobinas de peixe com relação à sensibilidade a $\mathrm{pH}$ e fosfatos inorgânicos, efeitos de temperatura sobre ligação de oxigênio, grau de cooperatividade, sensibilidade ao ion cloro, afinidade de oxigênio e em sensibilidade à desnaturalização por sais e uréla.

$\mathrm{O}$ fato de que diferenças interespecíficas nas propriedade funcionais das hemoglobinas de peixes, na realidade, representam adaptações aos meios ambientais internos e externos, é sugerido pelas numerosas correlaçỏes que têm sido observadas entre as propriedades funcionais das hemoglobinas e variações nos ambientes das espécies (Krogh \& Leitch, 1919; Willmar, 1934; Hochachka \& Somero, 1971; Powers, 1974; Baines, 1975; Powers et al., 1978) . Assim, um estudo comparativo e elucidação de variação estrutural e funcional em hemoglobina entre espécies de peixes com requerimentos ecológicos diferentes deveria dar um modelo útil para considerar a evolução e divergência de proteinas numa espécie através do tempo à medida que os meios internos e externos sofrem modificações.

Este trabalho resume experimentos realizados na hemoglobina isolada do bagre Brachyplatystoma sp. Representa parte de um esforço maior para deduzir os padrões de adaptação entre as hemoglobinas de bagre em relação a diferenças físicas nos habitats dos peixes. Os bagres amazônicos são especialmente apropriados a este tipo de levantamento. Das duas mil espécies conhecidas de bagres, mil e duzentos são encontradas na América do Sul (Nelson, 1976), muitas delas na bacia amazônica; os tipos de habitats ocupados pelas diversas taxas cobrem uma faixa grande. Assim, adaptações das hemoglobinas em resposta a gradientes de pressão de seleção podem ser observadas dentro de um mesmo grupo. Brachyplatystoma foi escolhido dada a sua

( ${ }^{*}$ ) - Versão original inglesa publicada em Comp. Bioch em. Physiol. vol. 62A (1). 1979.

(1) Department of Zoology, Duke University Marine Laboratory, Beaufort, North Carolina 28516.

(2) - CNR Center for Molecular Biology, Institute of Chemistry and Biochemistry, Faculty of Medicine, University of Rome, Italy.

(3) - Department of Zoology, University of Texas at Austin, Austin, Texas 78712

(4) - Department of Biology, John Hopkins University, Baltimore, Maryland 21218. 
disponibilidade, homogeneidade molecular e preferências de habitat, sendo que é um peixe ativo encontrado em águas de fluxo rápido.

\section{MATERIAIS E MÉTODOS}

\section{ISOLAMENTO}

Indivíduos de Brachyplatystoma sp. foram coletados em novembro e dezembro de 1976, durante uma expedição no R/V "Alpha Helix" no rio Solimões, uns $50 \mathrm{~km}$ rio acima da união deste com o Negro para formar o Ámazonas. Os peixes foram capturados por malhadeira de guelras e transportados ao "Alpha Helix" para estudo. O sangue foi obtido por punção cardíaca com seringas de vidro heparinizadas e frias, $(100 \mu)$ de heparina de sódio $(5000$ I.U $/ \mathrm{ml}$.) em $1,7 \% \mathrm{NaCl}$ por $5 \mathrm{ml}$ de sangue). Os eritrócitos foram lavados três vezes em dez volumes de Tris ImM frio, $\mathrm{pH} 8,01,1,7 \% \mathrm{NaCl}$ e então lisados em 3 volumes de Tris $1 \mathrm{mM}$, $\mathrm{pH} 8,0$ durante uma hora a $0^{\circ} \mathrm{C}$. Um décimo de volume $\mathrm{NaCl}$ IM foi agregado ao hemolisado e a mistura foi centrifugada a $28000 \times$ g por 15 minutos para remover o lixo celular. 0 sobrenadante foi deprovisto de sais e fosfatos orgânicos passando o material através de uma coluna de $2,5 \times 50 \mathrm{~cm}$ de resina de Sephadex G-25 equilibrada em Tris $0,1 \mathrm{mM}$, pH 8,5 seguido por um tratamento em coluna de dionificação contendo as seguintes resinas de cima para baixo: $2 \mathrm{~cm}$ Dowex-50 W forma amônio, $2 \mathrm{~cm}$ Dowex-1 forma acetato, $20 \mathrm{~cm}$ de resina de camada mista Bio. Rad AG 501-X8(D). A hemoglobina purificada foi guardada a $5^{\circ} \mathrm{C}$ até requerido. Uma amostra de hemolisado foi congelado a $-70^{\circ} \mathrm{C}$ e transportada em gelo seco a Beaufort, N. C. e logo guardada a $-20^{\circ} \mathrm{C}$ por dois meses após o que o hemolisado foi descongelado e usado em experimentos de filtração de gel.

\section{ELECTROFORESE}

Foi realizada electroforese de gel de poliacrilamida vertical ( $\mathrm{pH} 8,9,7,5 \%$ gel) à temperatura ambiente de acordo com Davis (1964) e Ornstein (1964). As amostras de hemoglobina $(1 \mathrm{mg} / \mathrm{ml})$ no tampão superior contendo 0,1 M $\beta$-mercaptoetano/ e ditionita foram borbuIhados com monóxido de carbono e aplicadas aos geis. Albumina de soro bovino foi usado como padrão. Os geis foram tingidos durante três horas em Azul Brilhante Coomassie R a $0,25 \%$ em uma solução de ácido acético, metanol absoluto e água (1:2:4) e decoloridos por difusão.

1. ABREVIAÇões: Bis-Tris, bis (2 hidroxietil) imino Tris (hidroximetil) metano; Tris, tris (hidroximetil) aminometano; ATP, adenosina trifosfato; EDTA, ácido etilano diamino tetraacético; I', constante de segunda ordem de velocidade de combinação de monóxido de carbono; K, constante de primeira ordem da velocídade de dessociação de oxigênio; $\mathrm{p} 1 / 2$, pressão parcial de oxigênio no qual a metade dos locus hemo estão ocupados.

\section{DETERMINAÇÃO DO PESO MOLECULAR}

Um experimento de filtração de gel com carboxi hemoglobina de Brachyplatystoma foi realizado de acordo com a técnica descrita por Martin et al. (1978c).

\section{ESTUDOS DE EQUILÍBRIO DE OXIGÊNIO}

Equilíbrios do sangue completo foram realizados a $30^{\circ} \mathrm{C}$ pelo método de Powers et. al. (1978) utilizando um analisador de dissociação de oxigênio Hem-O-Scan (American Instruments Corp.). Os equilíbrios de oxigênio de hemoglobina purificada foram realizados a $20^{\circ} \mathrm{C}$ como descrito por Riggs \& Wolbach (1956). As soluções de hemoglobina (60 $\mu \mathrm{M}$ hemo) foram levadas a uma concentração iônica de 0,05 em tampões Tris ou Bis-tris. Os valores $\mathrm{P}_{1 / 2}$ foram determinados como uma função do $\mathrm{pH}$. Os experimentos foram realizados com hemolisados "stripped" e hemolisados "stripped" + 1mM ATP.

\section{ANÁLISES CINÉTICAS}

Os experimentos cinéticos foram realizados com um aparelho de fluxo fechado GibsonDurrum do tipo originalmente descrito por Gibson \& Milnes (1964) equipado com uma faixa de observações de $2 \mathrm{~cm}$. As cinéticas de dissociação de oxigênio foram medidas pelo método da dithionita (Antonini \& Brunori, 1971) na presença e ausência de $1,25 \mathrm{mM}$ ATP. As concentrações hemo foram de 10-20 $\mu \mathrm{M}$ 
antes de misturar. A constante da velocidade de combinação de segunda ordem para o monóxido de carbono foi obtida por mistura rápida de soluções de hemoglobina dehidrogenadas e reduzidas $(10-20 \mu \mathrm{M}$ hemo) em tampões Tris ou Bis-tris de concentração iônica $0,1 \mathrm{com}$ uma solução de monóxido de carbono $86 \mu \mathrm{M}$ dissolvida em água. As soluções de monóxido de carbono foram obtidas por diluição quantitativa de soluções "stock" 0,86 mM de água destilada equilibrada com o gás a $1 \mathrm{~atm} e$ e $30^{\circ} \mathrm{Cl} \pm 1.9^{\circ} \mathrm{C}$. Os experimentos de combinação de $\mathrm{CO}$ foram realizados com soluções "stripped" ou soluções "stripped" com 12,5 $\mu \mathrm{M}$ ou 1,25 mM ATP como função do $\mathrm{pH}$. A reação foi estudada a uma concentração de $\mathrm{CO}$ e analisada como um processo de pseudo primeira ordem.

\section{TAMPÕES}

Tampões Tris foram usados em experimentos de $\mathrm{pH}$ acima de 7,0 sendo que tampões Bistris foram usados em experimentos de $\mathrm{pH}$ mais baixo. Nos experimentos com hemolisados as soluções de hemoglobina foram preparadas a concentração iônica de 0,05 . A concentração iônica das soluções de hemoglobina usadas nos experimentos cinéticos foi de 0,1 antes de misturas.

\section{Resultados}

\section{CARACTERIZAÇÃO DA HEMOGLOBINA}

DE BRACHYPLATYSTOMA

Em contraste com as hemoglobinas de outros membros do Pimeloididae (ex. Pimelodella sp; Pimelodus sp.) a hemoglobina de Brachyplatystoma migra como uma espécie molecular simples em electroforese de gel de disco e de foco isoelétrico (Bunn \& Riggs, 1978; Fyhn ot al., 1978). Os estudos de filtração de gel indicam que a CO-hemoglobina é tetramerica a $\mathrm{pH} 8,0 \mathrm{com}$ um peso molecular aparente de 69000 .

\section{EQUILÍBRIOS DE OXIGÊNIO}

A curva de oxigenação do sangue completo de B Pachyplatystoma a $30^{\circ} \mathrm{C}$ aparece na Fig. 1 . A diferença na posição das duas curvas medida na ausência e presença de $5,6 \% \mathrm{CO}_{2}$, res- pectivamente, ilustra o grande efeito Bohr desta hemoglobina. $O \mathrm{P}_{1 / 2}$ sem $\mathrm{CO}_{2}$ é $10,7 \mathrm{~mm}$ $\mathrm{Hg}$ e com $5,6 \% \mathrm{CO}_{2}$ é de $25,1 \mathrm{~mm} \mathrm{Hg}$. A forma das duas curvas indica ligação cooperativa de oxigênio. $\mathrm{O}$ valor $n$ do sangue sem $\mathrm{CO}_{2}$ é 2,1 e o valor $n$ do sangue com $5,6 \% \mathrm{CO}_{2}$ é de 1,3 .

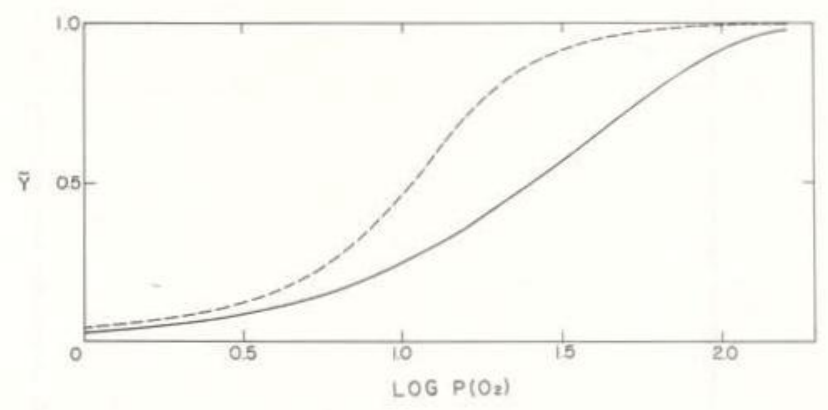

Fig. 1 - Curva de oxigênio do sangue completo heparinizado de Brachyplatystoma sp a $30^{\circ} \mathrm{C}$. As curvas $1(-$.$) e 2$ (-) representam de $5,6 \% \mathrm{CO}_{2}$ res. pectivamente.

Os resultados dos experimentos de equilibrio de oxigênio com hemoglobina "stripped" são apresentados na Fig. 2. Um efeito Bohr alcalino é aparente entre $\mathrm{pH} 8,4$ e 5,9. O efeito Bohr medido entre pH 7,0 e 8,0 para a hemoglobina "stripped" é $\Delta \log \mathrm{P}_{1 / 2} / \Delta \mathrm{pH}=-0,23$. Este efeito é aumentado agregando $1 \mathrm{mM} \mathrm{ATP}$, $\Delta \log \mathrm{P}_{1 / 2} / \Delta \mathrm{pH}=-0,58$. O ATP diminui a a afinidade de oxigênio a valores de $\mathrm{pH}$ menores que 9,0 sendo que a diminuição máxima ocorre perto de $\mathrm{pH} 7,0$. Perto de $\mathrm{pH} 8.0$ o $\mathrm{p} 1 / 2$, aumenta aproximadamente duas vezes na presença de ATP; a pH 7,0, a mudança é de mais ou menos 6 vezes. A curva de dependência de $\mathrm{pH}$, em experimentos nos quais há adição de ATP, é corrida uma unidade de $\mathrm{pH}$ à direita a valores baixos de $\mathrm{pH}$. Abaixo de pH 6,0 um efeito Bohr ácido ocorre em solução de Hemoglobina "stripped". Adição de $1 \mathrm{mM}$ ATP leva o efeito Bohr ácido a valores mais altos de pH. Não há efeito Root evidente a pH baixo.

A cooperatividade de hemoglobina também varia em função do $\mathrm{pH}$; valores de $n$ variando de 1 a pH 5,4 até 1,7 a pH 7,0 foram obtidos em soluções "stripped". A valores de $\mathrm{pH}$ acima de 7,0 o valor de $n$ diminui; a $\mathrm{pH} 9,0 \mathrm{n}=1,3$. A presença de ATP indica um aumento de $n$ 
a valores de $\mathrm{pH}$ acima de 7,0 e até $\mathrm{pH}$ de 8,06 . Entre $\mathrm{pH}$ de 5,9 e 8,06 na presença de $1 \mathrm{mM}$ ATP, $n$ varia de 1,2 a 2 . Acima de $\mathrm{pH} 8.06$ o valor de $n$ diminui e a $\mathrm{pH} 9,0$ é igual ao obtido para a solução de hemoglobina "stripped".

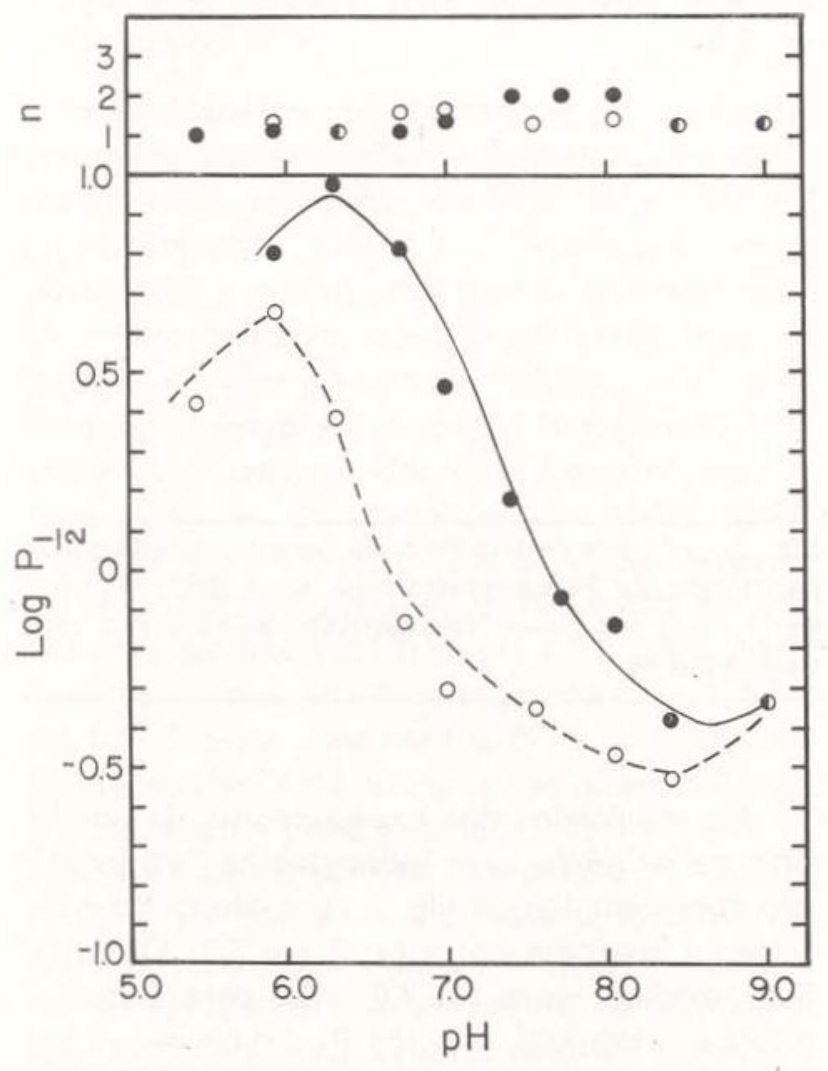

Fig. 2 - Gráfico do $\log \mathrm{P} 1 / 2$ e $\mathrm{n}$ de hemoglobina "stripped" de Brachyplatystoma vs. pH. Hemoglobina "stripped" (O) e "stripped" + ImM ATP (•). Condições como descritas no texto.

O $P_{1 / 2}$ de hemoglobina "stripped" é de $0,45 \mathrm{~mm} \mathrm{Hg}$ a $\mathrm{pH} 7,4 ;$ o $P_{1 / 2}$ é de $1,25 \mathrm{~mm}$ Mg após adição de $1 \mathrm{mM}$ ATP. Estas afinidades de oxigênio são consideravelmente maiores que os valores reportados acima para sangue completo. Esta discrepância também ocorre em outros trabalhos (Martin et al., 1978a e 1978b) e é provavelmente devida às diferenças em $n$ as temperaturas experimentais. Por exemplo, Powers et al. (1978) encontraram que o $\mathrm{P}_{1 / 2}$ de eritrócitos isolados de Pterygoplichthys sp. (outro bagre amazônico) mudou de 2,4 a $10,7 \mathrm{~mm} \mathrm{Hg}$ quando a temperatura experimental foi alterada de 20 a $30^{\circ} \mathrm{C}$. Prosser (1973) notou que o $P_{1 / 2}$ da hemoglobina de peixes geralmente aumenta em $1 \mathrm{~mm} \mathrm{Hg}$ por cada grau de aumento na temperatura. A alta afinidade das soluçōes de hemoglobina contendo ATP também podia ser atribuída, nestes experimentos, a ausência de guanosina trifosfato e inositol hexafosfato, cada um dos quais é mais efetivo que ATP em diminuir a afinidade de oxigênio da hemoglobina em certas outras espécies de peixe (Tan \& Noble, 1973; Torracca et al., 1977). A pH 9,0 o $\mathrm{P}_{1 / 2}$ da hemoglobina "stripped" é de $0,46 \mathrm{~mm} \mathrm{Hg}$. Uma pequena queda em $\mathrm{P}_{1 / 2}$ ocorre entre $\mathrm{pH} 9,0$ e 8,4 até 0,28 $\mathrm{mm} \mathrm{Hg}$. Este efeito também foi notado em estudos de outra espécie de bagre Hoptosternum littorale por Garlick et al. (1978). Entre pH 8,4 e 5,9 o $P_{1 / 2}$ aumenta 16,2 vezes indo de 0,28 até $4,55 \mathrm{~mm} \mathrm{Hg}$. Após adições de ATP a mudança de $\mathrm{P}_{1 / 2}$ aumenta até 23 vezes indo de 0,42 $\mathrm{mm} \mathrm{Hg}$ a pH 8,4 até $9,55 \mathrm{~mm} \mathrm{Hg}$ a pH 6,4.

\section{CINÉTICAS DE LIGAÇÃo DE LIGANDOS}

As cinéticas de dissociação de oxigênio da hemoglobina de Brachyplatystoma são homogêneas a todos os $\mathrm{pH}$ tanto com e sem 1,25 $\mathrm{mm} \mathrm{Hg}$. A constante de taxa de primeira ordem é dependente tanto do $\mathrm{pH}$ quanto do ATP (Flg. 3). Um aumento na taxa de 2,3 vezes ocorre entre $\mathrm{pH} 8,3$ e 5,95 variando até um aumento de 3,75 vezes na presença de ATP. Na ausência de ATP, a taxa muda de $20 \mathrm{seg}^{-1}$ a $50 \mathrm{seg}^{-1}$ entre $\mathrm{pH} 8,0$ e 5,25 sendo que adições de ATP causam um aumento na taxa de dissociação até aproximadamente $73 \mathrm{seg}^{-1}$ a pH 5,95. A $\mathrm{pH} 8,2$ o ATP não muda a taxa de dissociação. A pH 5,0 uma diminuição na taxa de dissociação ocorre nas soluções de hemoglobina tratadas com ATP não "stripped", o que é expressão cinética de efeito Bohr ácido. O efeito Bohr alcalino cinético é semelhante ao descrito para outras espécies de teleósteos. Porém, a magnitude da mudança de taxa entre $\mathrm{pH}$ 5,95 e 8,0 é menor que a observada para outras hemoglobinas de teleósteos. Por exemplo, a hemoglobina de Pseudodoras (Martin et al., 1978b) que em muitos aspectos é bem semeIhante a de Brachyplatystoma sp. apresenta mudanças na taxa de dissociação de 7 vezes na ausência e 10 vezes na presença de ATP porém, as afinidades absolutas de oxigênio a $\mathrm{pH}$ alto e baixo e as mudanças relativas das afinidades são similares nestas hemoglobinas quando em presença de ATP. Isto é possível 
face à taxa de combinação de ligandos extremamente rápida da hemoglobina de Pseudodoras. Os resultados dos experimentos cinéticos, apoiam as conclusões dos experimentos de equilíbrio em que as taxas cie dissociação indicam efeitos Bohr tanto ácido quanto alcalinos.

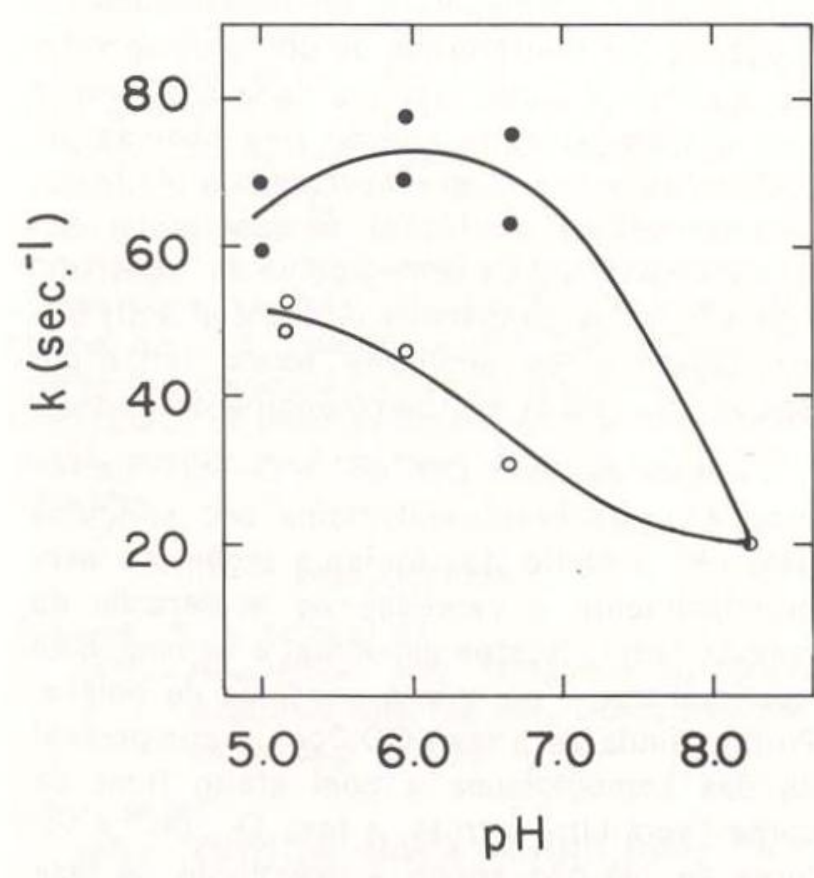

Fig. 3. - Dependências do pH da constante de dissociação de primeira ordem, $\mathbf{K}$, para oxigênio calculada dos dados de velocidade inicial. Condições como descritas no texto. Hemoglobina "stripped" (O); "stripped" + IMM ATP (•).

\section{CINÉTÍCAS DE COMBINAÇÃO DE CO}

A reação de combinação de $\mathrm{CO}$ foi estudada a uma concentração de $\operatorname{CO}\left(4,15 \times 10^{-5} \mathrm{M}\right)$ como função do $\mathrm{pH}$ e da concentração de ATP e analisada como um processo de pseudo primeira ordem. Os resultados são resumidos na Fig. 4. A reação é homogênea a $\mathrm{pH} 8,3$, porém virando autocatalítica a $\mathrm{pH} 7,0$, o que é indicativo de interações cooperativas entre as subunidades. A pH 7,0 a taxa inicial de reação é aproximadamente a metade da observada ao nível de metade de saturação. A constante de taxa inicial de segunda ordem é dependente do $\mathrm{pH}$ aumentando de $2 \times 10^{5} \mathrm{M}^{-1}$ seg-1 $^{-1}$ a $6,7 \times 10^{5}$ $\mathrm{M}^{-1}$ seg $^{-1}$ entre pH 5,9 e 8,3 em soluções de hemoglobina sem $1 \mathrm{mM}$ ATP.

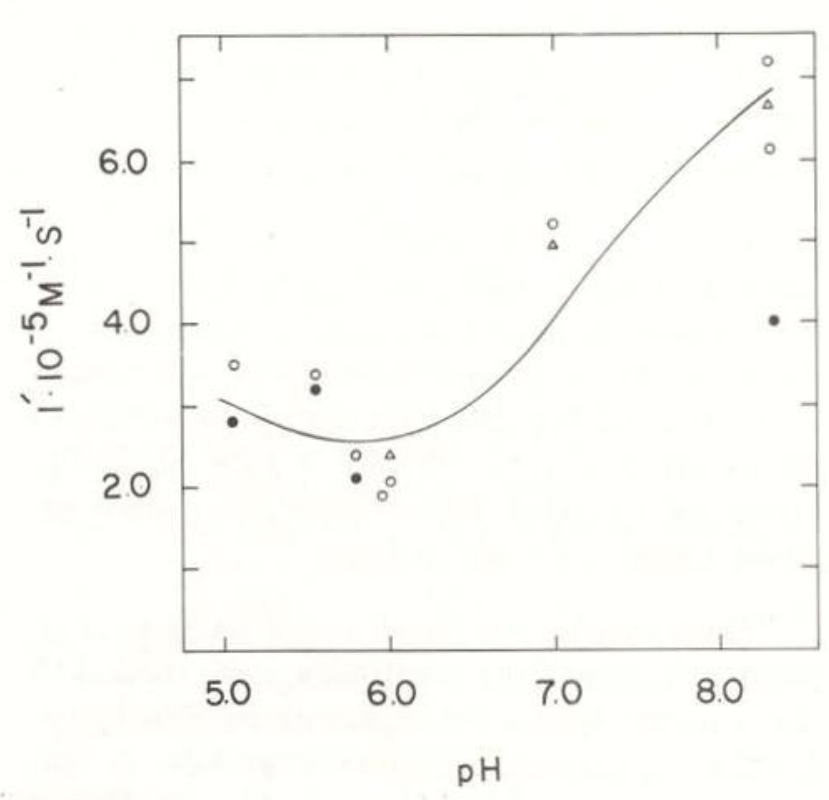

Fig. 4-Constante de velocidade de combinação de segunda ordem para ligação de $\mathrm{CO}, 1^{\prime}$, vs. pH. A reação é autocatalitica abaixo de pH 8,3 e os valores reportados representamos a velocidades iniciais de reação. Condições como descritas no texto. Hemoglobina "stripped" (O), "stripped" + 12,5 uM $\operatorname{ATP}(\Delta)$, "stripped" + $125 \mathrm{~m} \operatorname{ATP}(\bullet)$.

Há considerável variação nos dados de combinação de $\mathrm{CO}$ o que dificulta a interpretação com relação aos efeitos do ATP, porém a presença de $1,25 \mathrm{mM}$ ATP diminui ligeiramente a taxa $\mathrm{CO}$ " on" a pH 5,1 e a diminui grandemente a $\mathrm{pH} 8,3$. A direção da mudança nas constantes $\mathrm{CO}$ "on" e 02 "off" com o pH estão de acordo com a variação observada no $\mathrm{p} 1 / 2$ para o oxigênio em estudos de equilíbrio de oxigênio. As diferenças em magnitude da variação entre os estudos cinéticos e de equilíbrio podem surgir face aos efeitos diferenciais do $\mathrm{pH}$ no processo de combinação com $\mathrm{CO}$ e $\mathrm{O}_{2}$.

\section{Díscussão}

O sangue de Brachyplatystoma contem só um componente de hemoglobina, sendo que outros membros dos Pimelodidae (foram examinados espécies de seis gêneros) possuem componentes múltiplos da hemoglobina (Fyhn et al., 1978). Ainda dentro do gênero Brachyplatystoma foi descoberta uma espécie com componentes múltiplos. Fenômeno similar foi 
encontrado por Bonaventura et al. (1976) em Leiostomus xanthurus, única espécie de cinco examinada da família Sciaenidade que possui um sistema de hemoglobina simples.

Os bagres da família Pimelodidae possuem bexigas natatórias. Porém, como foi indicado por Farmer et al. (1978), não possuem os dois sistemas multiplicadores contra-corrente que vários peixes usam para secreção de oxigênio, a rete mirabile e rete choroide. Todas as espécies examinadas por estes autores também de efeito Root.

Experimentos realizados em sangue completo e hemoglobina purificada com $1 \mathrm{mM}$ ATP demonstram que a hemoglobina de Brachypiatystoma apresenta efeito Bohr grande: A log $\mathrm{p} 1 / 2 / \mathrm{ApH}=-0,58$ para soluçōes de hemoglobina mais $1 \mathrm{mM}$ ATP em comparação com os dados das hemoglobinas de outras espécies de bagre, (ex, Hoplosternum littorale, $-0,33$, solução de hemoglobina não fracionada cơm $1 \mathrm{mM}$ ATP, Garlick et al., 1978; Pseudodoras sp., $-0,14$, solução de hemoglobina mais $1 \mathrm{mM}$ ATP, Martin et al., 1978; Prerygoplichtys sp, $-0,31$, sangue completo, Johansen \& Magnum, 1978; Ictalurus nebulosus - 0,31 , sangue completo, Grigg, 1969) . Brachyplatystoma sp. é a espécie siluróide mais ativa das enumeradas acima, sendo freqüentemente encontrada nos centros de rios de águas rápidas (Fink \& Fink, 1978). Reischl (1977) examinou a afinidade de oxigênio da hemoglobina de outra espécie pimelodidae, Pimelodus maculatus na presença de $600 \mathrm{mM}$ ATP e encontrou um efeito Bohr de $-0,56$ entre $\mathrm{pH} 7,0$ e 8,0 . Powers et al. (1978) descobriram que $\mathrm{CCO}_{2}$ tem maior efeito na afinidade de oxigênio dos eritrócitos de bagres das famílias Pimelodidae e Auchenipleridae que apresentam espécies de movimento rápido. Também observaram que $0 \mathrm{CO}_{2}$ diminui em grau menor a afinidade de oxigênio do sangue de bagres das famílias Loricariidae e Callichthyidae que são geralmente peixes bênticos vagarosos encontrados nas margens de riachos. Esta tendência na magnitude do efeito Bohr tem sido notada por Riggs (1970) para uma variedade de peixes e esta presumivelmente correlata com o grau de necessidade para suprimento rápido de oxigênio nos tecidos.
A hemoglobina de Brachyplatystoma é semelhante à hemoglobina de mamíferos em apresentar efeitos Bohr ácidos e alcalinos, efeito de fosfato e não apresenta efeito Root. Porém, a valores baixos de $\mathrm{pH}$ a cooperatividade é reduzida com $\mathrm{n}=1$ a $\mathrm{pH} 5,4$. Valores baixos de $n$ podem resultar da heterogeneidade inter ou intramolecular de ligação de oxigênio sendo que a hemoglobina foi considerada ho mogênea por eletroforese de gel, o baixo valor de $n$ provavelmente aparece dada a diferença nas propriedades de ligação das cadeias semelhantes a $\propto$ e $\beta$ que aparecem a pH baixo. Bonaventura et al. (1976) demonstraram que as cadeias $\propto$ e $\beta$ da hemoglobina de "spotfish" ligam ligandos de maneira diferencial a pH baixo. Observações similares foram feitas por Tan et al. (1973) em hemoglobina de carpa.

Ambas as taxas CO "on" e $\mathrm{O}_{2}$ "off" da hemoglobina de Brachyplatystoma são atingidas pelo $\mathrm{pH}$; o efeito dos fosfatos orgânicos está principalmente e expresso na aceleração da taxa $\mathrm{O}_{2}$ "off". Nestes aspectos, a hemoglobina assemelha-se a de outras espécies de peixes. Porém, ainda se a taxa $\mathrm{CO}$ "on" é comparável às das hemoglobinas e com efeito Root de carpa "spotfish" e truta, a taxa $\mathrm{O}_{2}$ "off" a valores de $\mathrm{pH}$ não atinge a magnitude da taxa observada nessas mesmas hemoglobinas.

\section{AgRADECIMENTOS}

Maurizio Brunori agradece ao Conselho Nacional de Pesquisas da Itália pelo apoio financeiro. Queremos expressar nosso agradecimento ao Governo Brasileiro pela generosa colaboração durante todas as fases da expeção do "Alpha Helix". Apoio adicional foi dado pelo contrato ONR ND OOIH-75-C-0190 (J.B.), Bolsa NSF DEB 76-19877 (D.A.P.), National Geographic Society (D.A.P.) e bolsa NIH 21314 (a A. Riggs).

\section{SUMMARY}

The single hemoglobin component of Brachy. platystoma sp. has been isolated. The CO-hemoglobin has an apparent molecular weight of 69,000 as determined by gel filtration. The hemoglobin displays both acid and alkaline Bohr effects, an organic phosphate effect and no Root effect. The whole blood $p_{1 / 2}$ for oxygen shifts from $10.7 \mathrm{~mm}$ 
$\mathrm{Hg}$ in air equilibrated solutions to $25.1 \mathrm{~mm} \mathrm{Hg}$ after the addition of $5.6 \% \mathrm{CO}_{2}$ to the equilibration gas. The $p_{1 / 2}$ of purified hemoglobin varies from $0.3 \mathrm{~mm} \mathrm{Hg}$ at pH 8.4 to $4.5 \mathrm{~mm} \mathrm{Hg}$ at $\mathrm{pH} 5.9$. The Bohr effect measured for stripped hemoglobin between $\mathrm{pH} 8.0$ and 7.0 is $\Delta \log \mathrm{p}_{1 / 2} / \Delta \mathrm{pH}=-0.23$. Additions of $1 \mathrm{mM}$ ATP induce a shift in the Bohr effect to $\Delta \log p_{1 / 2} / \Delta \mathrm{pH}=-0.58$ over the same $\mathrm{pH}$ range. The $\mathrm{n}$ value of stripped hemoglobin solutions varies from 1 at $\mathrm{pH} 5.9$ to 1.7 at $\mathrm{pH} 70$. Additions of $1 \mathrm{mM}$ ATP shift the variation in $\mathrm{n}$ to higher $\mathrm{pH}$ values, and causes an increase in the $\mathrm{n}$ value, $\mathrm{n}=2$ at $\mathrm{pH}$ 7.4. The kinetics of carbon monoxide binding and oxygen dissociation are $\mathrm{pH}$ dependent. The $\mathrm{CO}_{\text {on }}$ rate becomes autocatylytic as the $\mathrm{pH}$ is lowered, indicating positive subunit interactions. The $\mathrm{O}_{2}$ off rate was homogeneous at all $\mathrm{pH}$ values. The Bohr effects of Brachyplatystoma hemoglobin and other pimelodid hemoglobins are greater than those determined for the unfractionated hemoglobins of more sedentary species from other catfish families such as the Loricariidae and Callichthyidae.

\section{BIBLIOGRAFIA}

ANTONINI, E. \& BRUNORI, M.

1971 - Hemoglobin and Myoglobin in Their Reactions with Ligands. North Holland, Amsterdam. $436 \mathrm{pp}$

BAINES, G.W.

1975 - Blood pH effects in eight fishes from the teleostian family Scorpaenidae. Comp. Biochem. Physiol. 51, 833-849.

Bonaventura, C.; Sullivan, B.; Bonaventura, J. \& BRUNORI, M.

1976 - Spot hemoglobin: studies on the Root effect hemoglobin of a marine teleost. J. Biol. Chem. 251, 1871-1876.

BRIDGE, J.W. \& HADDON, G.C.

1893 - Contributions to the anatomy of fishes. II. The air bladder and Weberian ossicles in the siluroid fishes. Phil. Trans. Soc. (B) 184, 65-434.

BUNN, H.F. \& RIGGS, A.

1978 - A medida do efeito Bohr em hemoglobinas de peixe por focalização elétrica em gel. Acta Amazonica 8(4) : Suplemento. (Este volume).

DAvis, B.J.

1964 - Disc electrophoresis - II. Method and application to human serum proteins. Ann. N. Y. Acad. Sci. 121, 404-427.

FARMER, M.; FYHN, H.J.; FYHN, U.E.H. \& NOBLE, R.W. 1978 - Ocorrência de hemoglobinas de eferto Root em peixes amazônicos. Acta Ama. zonica 8(4): Suplemento. (Este volume)
FINK, W.L. \& FINK, S.V.

1978 - A Amazônia Central e seus peixes. Acta Amazonica 8(4) : Suplemento. (Este volume).

FYhN, U.E.H.; FyhN, H.J.; DAVIS, B.J.; POWERS, D.A.; FINK, W.L. \& GARLICK, R.L.

1978 - Heterogeneidade de hemoglobina nos peixes da Amazônia. Acta Amazonica 8(4): Suplemento. (Este volume).

GARLICK, R.L.; BUNN, H.F.; FYhN, U.E.H.; MARTIN, J.P.; Noble, R.W. \& Powers, D.A.

1978 - Estudos funcionais dos componentes separados da hemoglobina de um bagre de respiração aérea, Hoplosternum littorale. (Hancock). Acta Amazonica 8(4): Suplemento. (Este volume).

GIBSON, Q.H. \& MILNES, L.

1964 - Apparatus for rapid and densitive spectrophotometry. Biochem. J. 91, 161-171.

Grigo, G.C.

1969 - Temperature-induced changes in the oxygen equilibrium curve of the blood of the brown bullhead, Ictalurus rebulosus. Comp. Biochem. Physiol, 28, 120-1223.

HochachKa, P. \& SOMERo, G.

1971 - Biochemical adaptation to the environment. In: Fish Physiology vol. VI pp. 99-156 (Hoar, W.S. \& Randall, eds.) Academic Press, New York.

Johansen, K. \& MANGuM, C.P.

1978 - Respiratory properties of the blood of Amazon fishes. J. Exp. Zool. (In press).

KROGH, A. \& LeITVH, I.

1919 - The respiratory function of the blood in fishes. J. Physiol. Lond. 52, 288-300.

Martin, J.P.; Bonaventura, J.; BRUnori, M.; Fyhn, H.J.; Fyhn, U.E.H.; Garlick, R.L.; Powers, D.A. \& WILSON, M.T.

1978 - Isolamento e caracterização dos componentes de hemoglobina de Mylossoma sp., um teleósteo da Amazônia. Acta Amazonica 8(4) : Suplemento. (Este volume).

Martin, J.P.; BonaVentura, J.; FYhN, H.J.; FYhN

U.E.H.; Garlick, R.L. \& Powers, D.A.

1978 - Estudo da estrutura e função das hemoglobinas isoladas das arraias da Amazônia do gênero Potamotrygon. Acta Amazonica 8(4): Suplemento. (Este volume).

Martin, J.P.; FYhn, H.J.; FYhn, U.E.H.; Garlick, R.L. \& POWERS, D.A.

1978 - A hemoglobina do Pseudodoras. Um "bagre" da América do Sul: isolamento, caracterização e estudos dos ligantes. Acta Amazonica 8(4): Suplemento. (Este volume). 
NeLSON, J.A.

1976 - Fishes of the World. Wiley-Interscience, New York, $416 \mathrm{pp}$.

ORNSTEn, L.

1964 - Disc electrophoresis - I. Background and theory. Ann. N. Y. Acad. Sci. 121, 321-349.

POWERS, D.A.

1974 - Structure, function and molecular ecology of fish hemoglobins. Ann. N. Y. Acad. Sci. 241, 472-490.

POWers, D.A.; FYHN, H.J.; FYhN, U.E.H.; MARTIN, J.P.; GARLICK, R.L. \& WOOD, S.C.

1978 - Estudo comparativo de equilíbrio de oxigênio no sangue de 40 gêneros de peixes da Amazônia. Acta Amazonica 8(4): Suplemento. (Este volume).

Prosser, C.L.

1973 - Comparative Animal Physiology. 3rd ed. W. B. Saunders Company, Philadelphia. $966 \mathrm{pp}$.

REICHIIN, M. \& DAVIS, B.J.

1978 - Relações antigênicas entre peixes comuns à bacia do rio Amazonas. Acta Amazonica 8(4) : Suplemento. (Este volume).

REISCHI, E.

1977 - Oxygen equilibria of the hemoglobins from the fresh water catfish Pimelodus maculatus (Lacepede, 1803). Comp. Biochem. Physiol. 58A, 217-221.
RIGos, A.

1970 - Properties of fish hemoglobins. In: Fish Physiology (Hoor, W.S. \& D.J. Randall, eds.) Vol. IV, pp. 209-251. Acade. mic Press, New York.

Rigos, A. \& Wolbach, R.A.

1956 - Sulfhydryl groups and the structure of hemoglobin. J. Gen. Physiol. 39, 585-605.

TAN, A.L. \& NOBLE, R.W.

1973 - The eftect of inositol hexaphosphate on allosteric properties of carp hemoglobin. J. Biol. Chem. 248, 7412-7416.

TAN, A.L.; Noble, R.W. \& Gibson, Q.H.

1973 - Conditions restricting allosteric transitions in carp hemoglobin. J. Biol. Chem. 248, 2880-2888.

TORRACA, A.M.; RASchetti, R.; SALVIOLA, R.; Ricciardi, G. \& WINTERHALTER, K.H.

1977 - Modulation of the Root effect in goldfish by ATP and GTP. Biochim. Biophys. Acta 496, 367-373.

WIMMER, E.N.

1934 - Some observations on the respiration of certain tropical fresh-water fish. J. Exp. Biol. 11, 282-30t. 